\title{
COMBINATION OF LOCAL DESCRIPTORS AND GLOBAL FEATURES FOR LEAF RECOGNITION
}

\author{
Maliheh Shabanzade ${ }^{1}$, Morteza Zahedi ${ }^{1}$ and Seyyed Amin Aghvami ${ }^{2}$ \\ ${ }^{1}$ Department of Computer Engineering and Information Technology, Shahrood \\ University Of Technology, Shahrood, Iran \\ Shabanzadeh@shahroodut.ac.ir, Zahedi@shahroodut.ac.ir \\ ${ }^{2}$ Department of Computer Engineering, Afarinesh University of Applied Science and \\ Technology, Shahrood, Iran \\ s_a_aghvami@yahoo.com
}

\begin{abstract}
Automatic leaf recognition system is a case coming to improve time-consuming and troublesome tasks which have mainly been carried out by botanists manually. This application as judged by common characteristics is popular in institutes for discovering new plant species, modernizing the management of botanical gardens and horticulture fields. In order to conduct a leaf recognition system, the features must be sufficiently distinctive to identify specific objects among many alternatives, where contain both local and global properties. So far, many researchers have represented some techniques which use local or global features only where face problems, such as many images are captured in different intensity, they are maybe sick or calamity, leaves have been damaged or cropped and so on. In this paper, a new method for leaf recognition system is proposed where both local descriptors and global features are employed, combined and finally the most discriminant features are selected by employing a linear discriminant analysis method. The experimental results show that using the feature vector containing the local features and global characteristics leads us to obtain $94.3 \%$ recognition rate.
\end{abstract}

\section{KEYWORDS}

Leaf Recognition, Image Processing, local descriptors, global features.

\section{INTRODUCTION}

Plants can be seen in many places, including roads, mountain paths, and fields, which have many different kinds where recognizing them can be useful in various applications. For instance, the institutes discovering new plant species need a process and method for plant recognition or management of botanical gardens. So far, this process is performed based on using leaves, and they should be examined with expert humans manually, where this operation is a time-consuming via a low-efficiency process.

In order to cope with the shortcomings of the introduced process which is done manually, current paper tries to design a system based on machine vision algorithms for improvement of result accuracy and reduction of process time. As the performance of such a recognition system depends on good feature selection, it is very important to select a set of features which can describe leaf characteristics very well. In this case, the other researchers have used a set of discriminative features including leaf shape, vein structure, teeth and color for recognition.

DOI : 10.5121/sipij.2011.2303 
Signal \& Image Processing : An International Journal (SIPIJ) Vol.2, No.3, September 2011

Common features break down into two categories, global features and local descriptors. The first category consists of the length and width of the leaf area or the other properties that define shape universalism, where most of the researchers in this area use a subset of these features [1-9]. Yanhua Ye presents the methods for computing global shapes of the leaves, such as aspect ratio and then leaf matching methods which can describe the overall shape of the leaves [10]. However, the contour is another important leaf property which is suitable factor for describing the teeth of the leaves. Counter shapes can be explained with centroid counter distance (CCD) method. The researches using CCD features for leaf recognition are reported in $[3,5,6,10]$.

The second category describing the leaf details involves texture based concept of the leaves where called local descriptors. Therewith, simple features in this matter are proposed by Gonzalez to obtain texture descriptors of any image, such as contrast, correlation, homogeneity [11]. Many researchers use a subset of texture features like the simple proposed features or similar ones [12-16] expecting these values are suitable factor for describing vein structure.

In opposite of the introduced benefits for the local descriptors and also global features, these features can not work alone for leaf recognition separately, assuming that two leaves have similar shape but different texture, or similar texture but many different shapes. Furthermore, a leaf recognition system using only one of the introduced features alone faces more problems listed as follow:

- The captured images from leaves have various properties in different conditions, such as yellow color in autumn, dried in winter or variation in intensity. These conditions lead to wrong calculations of correct local descriptors.

- Leaf shape may be incomplete, for example if animals eat leaves then some parts are cropped and shape properties change, so global features can not support this kind of problems well.

- Any leaf is capable for being sick or calamity, so many holes and dots occur in an image, thus local descriptors give unexpected values and cause mistake in recognition.

- There is no guarantee for the leaf in a taken image being with a normal size or desired angle in center of image, so it may cause a mistake certainly.

As it is explained, the local and global features are not able to help the recognition system achieving an acceptable results due to the wrong recognition in the different case of calamity, or changing the light conditions or seasons. Thus in this paper, we introduce a method for combination of the local descriptors and global features in order to cope with the shortcomings of each category of the features by using another one. The details about the method come in future sections.

\section{SYSTEM ARCHITECTURE}

Current paper presents an efficient approach consisting of following stages that usually used in machine vision systems:

- Pre-processing

- Feature extraction

- Classification

The detailed steps of the recognition system are described in next sections. Figure 1 shows the simplified block diagram that is proposed for a computer-aided leaf recognition system. As one can see the pre-processing and feature extraction stages start the process produce local descriptors 
Signal \& Image Processing : An International Journal (SIPIJ) Vol.2, No.3, September 2011

and global features. After combining the extracted features, in order to select the most discriminative features a linear discriminative analysis method is employed. Finally, the final feature vector hoping to be the best features containing local descriptors and global shape features is used by a statistical classifier.

\section{PRE-PROCESSING}

Normally, the leaf picture taken in an un-controlled environment which has to be used for a recognition system has some defects making it problematic for achieving a good recognition rate. Thus, before using a raw image, it is necessary to perform some pre-processing stages where a binarized image is necessary for extraction of global features and also a special segmentation process for extraction of local descriptors.

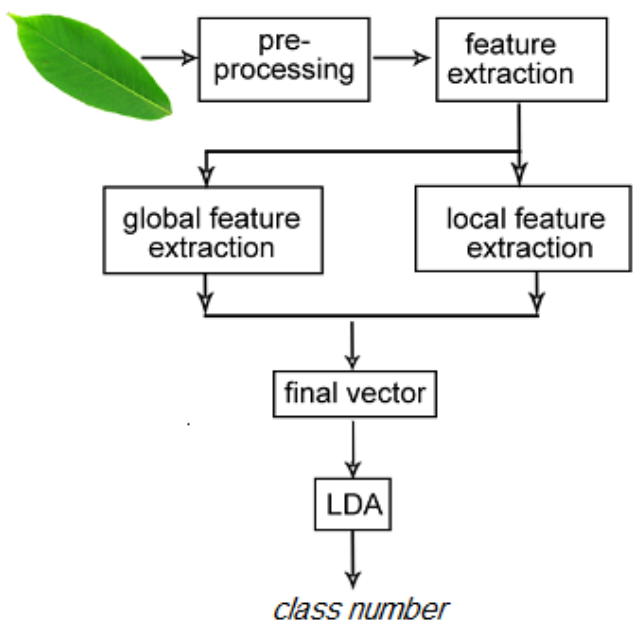

Figure1. System architecture for proposed method

In order to extract global features, after drawing the image histogram, an empirical threshold value leads us to a binarization process which produces a binary image where the background pixels are one and leaf area pixels are zero. This work is done with an adaptive thresholding method for each image in order to distinct the leaf from its background.

On the other hand, for extraction of local features, the leaf area containing the leaf part without background pixels is cropped. As leaf textures are symmetric in top and bottom parts, so segmentation of half area of a leaf is enough for describing the leaf features. With an aim to this purpose, we tierce the leaf width, and consequently get the middle part. Afterward, in order to use only the upper part, the leaf height is divided to the upper part and lower part. Eventually, several rows from top and bottom of the leaf are skipped in order to avoid using the leaf teeth. In next steps, a texture-based feature extraction is applied on the segmented image obtained from the preprocessing step.

\section{Feature Extraction}

The main work of a leaf recognition system is to extract common features among the images belonging to the same class in the images of the data set and consequently indexing them. The features are organized in two categories: local descriptors and global features.

Local features or texture based properties of a leaf in the image are six values that make a vector. On the other hand, global features describing universalism specification are calculated in a vector with four elements. Then, two vectors are concatenated making a feature vector with ten members which are named $\mathrm{f} 1, \mathrm{f} 2, \ldots$, and $\mathrm{f} 10$. 
Signal \& Image Processing : An International Journal (SIPIJ) Vol.2, No.3, September 2011

\subsection{LOCAL DESCRIPTORS}

This section focuses on the statistical texture-based descriptors of the leaf for indicating leaf properties. Gonzalez in [1] defines statistical moments and histogram-based features as the simplest methods for describing and presenting the leaf texture.

Measuring texture properties based on the only histogram, because of neglecting the information of pixel position regarding the other pixels, ignore and lose some useful information of the texture. In order to consider this kind of information, let $\mathrm{O}$ be a position operator that indicates relative position of two pixels, $f$ be an image with $L$ gray levels, $G$ be a matrix whose element $g_{i j}$ is the number of times that pixels with gray level $g_{i}$ occurs relative to the pixels with gray levels $\mathrm{g}_{\mathrm{j}}$, when the $\mathrm{O}$ operation is applied to the image $\mathrm{f}$. The matrix $\mathrm{G}$ is called co-occurrence matrix where its size is determined by the number of distinct gray levels in the input image.

Let $n$ be the total number of pair points in the image that satisfy $O$, so an estimation of the probability that a pair of pixels satisfying $O$ will have values $\left(g_{i}, g_{j}\right)$ is defined as follow:

$$
\mathrm{p}_{\mathrm{ij}}=\frac{\mathrm{g}_{\mathrm{ij}}}{\mathrm{n}}
$$

where sum of $p_{i j} s$ is one. In order to normalize $G$ and obtaining $G_{n}$, it is divided to $n$. However, each element of $G_{n}$ is equal to $P_{i j}$. Some other parameters needed for calculating the final features are listed as: $m_{r}$ is the average value of $G$ in rows, $m_{c}$ is the average value of $G$ in columns, $\sigma_{r}^{2}$ is the standard variance of $\mathrm{G}$ in rows and $\sigma^{2}{ }_{\mathrm{c}}$ in columns.

$$
\begin{gathered}
m_{r}=\sum_{i=1}^{k} i P(i) \\
m c=\sum_{j=1}^{k} j P(j) \\
\sigma_{r}^{2}=\sum_{i=1}^{k}\left(i-m_{r}\right)^{2} P(i) \\
\sigma_{c}^{2}=\sum_{i=1}^{k}\left(j-m_{c}\right)^{2} P(j) \\
\mathrm{P}(\mathrm{i})=\sum_{\mathrm{i}=1}^{k} \mathrm{P}_{\mathrm{ij}}
\end{gathered}
$$

Based on the above definitions, a set of useful descriptors regarding texture information are defined as follow:

Contrast is the contrast of the pixel and its neighbours calculated over all of the image pixels:

$$
f 1=\sum_{i=1}^{k} \sum_{j=1}^{k}(i-j)^{2} p_{i j}
$$

Correlation is the measure of correlation of a pixel with its neighbours over all of the image pixels:

$$
f 2=\sum_{i=1}^{k} \sum_{j=1}^{k} \frac{\left(i-m_{r}\right)\left(j-m_{c}\right) p_{i j}}{\sigma_{r} \sigma_{c}}
$$

Energy is sum of G elements:

$$
f 3=\sum_{i=1}^{k} \sum_{j=1}^{k} p_{i j}^{2}
$$

Homogeneity computes similarity of $\mathrm{G}$ to the diagonal matrix: 
Signal \& Image Processing : An International Journal (SIPIJ) Vol.2, No.3, September 2011

$$
f 4=\sum_{i=1}^{k} \sum_{j=1}^{k} \frac{p_{i j}}{1+|i-j|}
$$

Maximum probability gives an indication of the strongest response to O:

$$
f 5=\operatorname{Max}_{i, j}\left(p_{i j}\right)
$$

and Entropy shows randomization ratio in the image:

$$
f 6=-\sum_{i=1}^{k} \sum_{j=1}^{k} p_{i j} \log _{2}\left(p_{i j}\right)
$$

These are the feature values of $\mathrm{f} 1$ to $\mathrm{f} 6$ calculated from each image where they are called local descriptors.

\subsection{GLOBAL FEATURES}

Another stage for making the feature vector depending on generic characteristics of a leaf is the extraction of global features. Firstly, binary image is produced by employing an adaptive thresholding method; 1 value for background pixels and 0 for leaf area. Then, using the segmented leaf, the global features as a vector with 4 values are defined as follow:

Aspect ratio is the ratio between the maximum length $\mathrm{L}_{\max }$ and the minimum length $\mathrm{L}_{\min }$ of the minimum bounding rectangle around the leaf:

$$
f 7=\frac{L_{\max }}{L_{\min }}
$$

Rectangularity is the ratio between region of interest area $\left(\mathrm{A}_{\mathrm{ROI}}\right)$ and the aspect ratio:

$$
f 8=\frac{A_{R O I}}{f 7}
$$

Convex area ratio is defined as the ratio between $A_{R O I}$ and the convex hull area $\left(A_{c}\right)$ :

$$
f 9=\frac{A_{c}}{A_{R O I}}
$$

Circularity is defined based on the mean and variance of ROI:

$$
f 10=\frac{\mu_{R O I}}{\sigma_{R O I}}
$$

The above values are calculated for each image in order to make a 4-member feature vector (f7, f8, f9 and f10).

\section{LINEAR DisCRIMINANT ANALYSIS}

Linear discriminant analysis (LDA) is a technique for discriminating between two or more groups based on a series of variables. Let $\mathrm{X}$ be a $\mathrm{D}$-dimensional vector from one of $\mathrm{k}$ classes. Linear discriminant analysis can be used to classify $\mathrm{X}$ if the class is unknown. Briefly, Assume that $\mathrm{Y}$ is the final data in a projected space where $\mathrm{y}=\mathrm{w}^{\mathrm{T}} \mathrm{X}$. The scatter matrix is defined as:

$$
s_{i}=\sum_{x \in \text { class }_{i}}\left(x-\mu_{i}\right)\left(x-\mu_{i}\right)^{T}
$$

where $\mu_{\mathrm{i}}$ is the centroid of the $\mathrm{i}$-th class and $\tilde{\mu}_{i}$ is the centroid of projected data. Summing of $\mathrm{s}_{\mathrm{i}}$ known as within-class scatter matrix $\left(\mathrm{s}_{1}+\mathrm{s}_{2}+\ldots+\mathrm{s}_{\mathrm{k}}=\mathrm{s}_{\mathrm{w}}\right)$. In next step, calculate the scatter of the projection y: 
Signal \& Image Processing : An International Journal (SIPIJ) Vol.2, No.3, September 2011

$$
\begin{gathered}
\tilde{S_{i}}{ }^{2}=\sum_{y \in \text { class }_{i}}\left(y-\tilde{\mu}_{i}\right)^{2}=\sum_{x \in \text { class }_{i}}\left(W^{T} x-W^{T} \mu_{i}\right)=W^{T} S_{i} W \\
{\tilde{S_{1}}}^{2}+\ldots+{\tilde{\mathrm{S}_{K}}}^{2}=W^{T} S_{W} W
\end{gathered}
$$

Then difference between the projected means should be computed. Below formula shows this matter for two classes:

$$
\left(\tilde{\mu}_{1}-\tilde{\mu}_{2}\right)^{2}=\left(W^{T} \mu_{1}-W^{T} \mu_{2}\right)^{2}=W^{T}\left(\mu_{1}-\mu_{2}\right)^{T} W=W^{T} S_{B} W
$$

The matrix $\mathrm{s}_{\mathrm{B}}$ is known as between-class scatter matrix. LDA computes results by minimizing the $\mathrm{s}_{\mathrm{W}}$ and maximizing the $\mathrm{s}_{\mathrm{B}}$, thus achieving maximum class discrimination. For access this purpose and Estimate the parameters of the discriminating function, finally Fisher criterion is needed:

$$
w^{*}=\arg \max _{W}\left\{\frac{W^{T} S_{B} W}{W^{T} S_{W} W}\right\}
$$

\section{EXPERIMENTAL RESULTS}

The proposed method is developed in Matlab 2010 for verifying the validity of the model. In order to evaluate our algorithm objectively and compare with other methods, the proposed method is examined on 60 different plant species with 20 samples in each class. Those leaves are collected in the campus of Nanjing University and Sun Yat-Sen arboretum, China [4]. They are common plants in Yangtze Delta, China where many researches work on it. The database consists of various kinds of leaves in different categories so leads to universalism in system. Figure 2 shows an example of plant leaf images for each class.

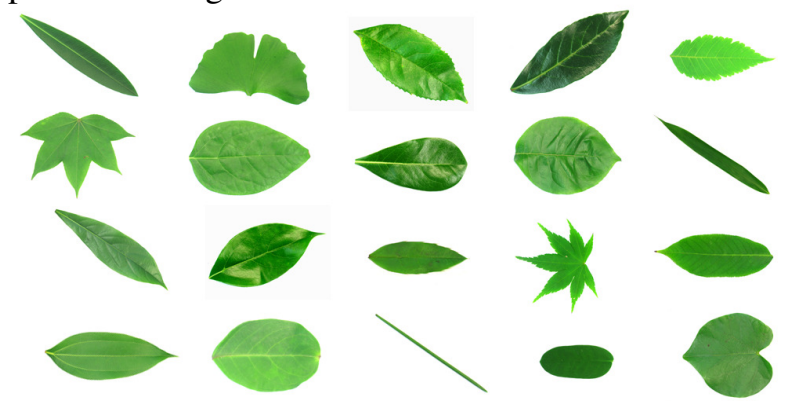

Figure 2. Some examples from the leaves dataset

In order to conduct the experiments, first of all the testing and training data sets are randomly picked up from the others, then local descriptors and global features are extracted from the sample images before applying the classification algorithm.

For clarification details of the extracted features and benefits of them for current subject, two diagrams are shown in Figure 3. In each diagram, considering a color that shows a class points for the corresponding feature, if class variance for a set of color points is low and center of the points is located in a large distance from the other class means, it illustrates that the feature is suitable and discriminative. In other words, range of any feature value for each class should not be so much overlapped in diagrams.

In Figure 3, the diagrams show that the third and fifth features (f3, f5) are distinct for different classes, so it is expected that they can lead to a better classification result. Horizontal axis is class value that consists of 20 classes, and vertical axis shows different values of $\mathrm{f} 3$ (in next diagram 
Signal \& Image Processing : An International Journal (SIPIJ) Vol.2, No.3, September 2011

f5) for each class value. Other features extracted from the image samples have similar diagrams where combining all of the features helps us to obtain a good feature vector which is efficient for classification.
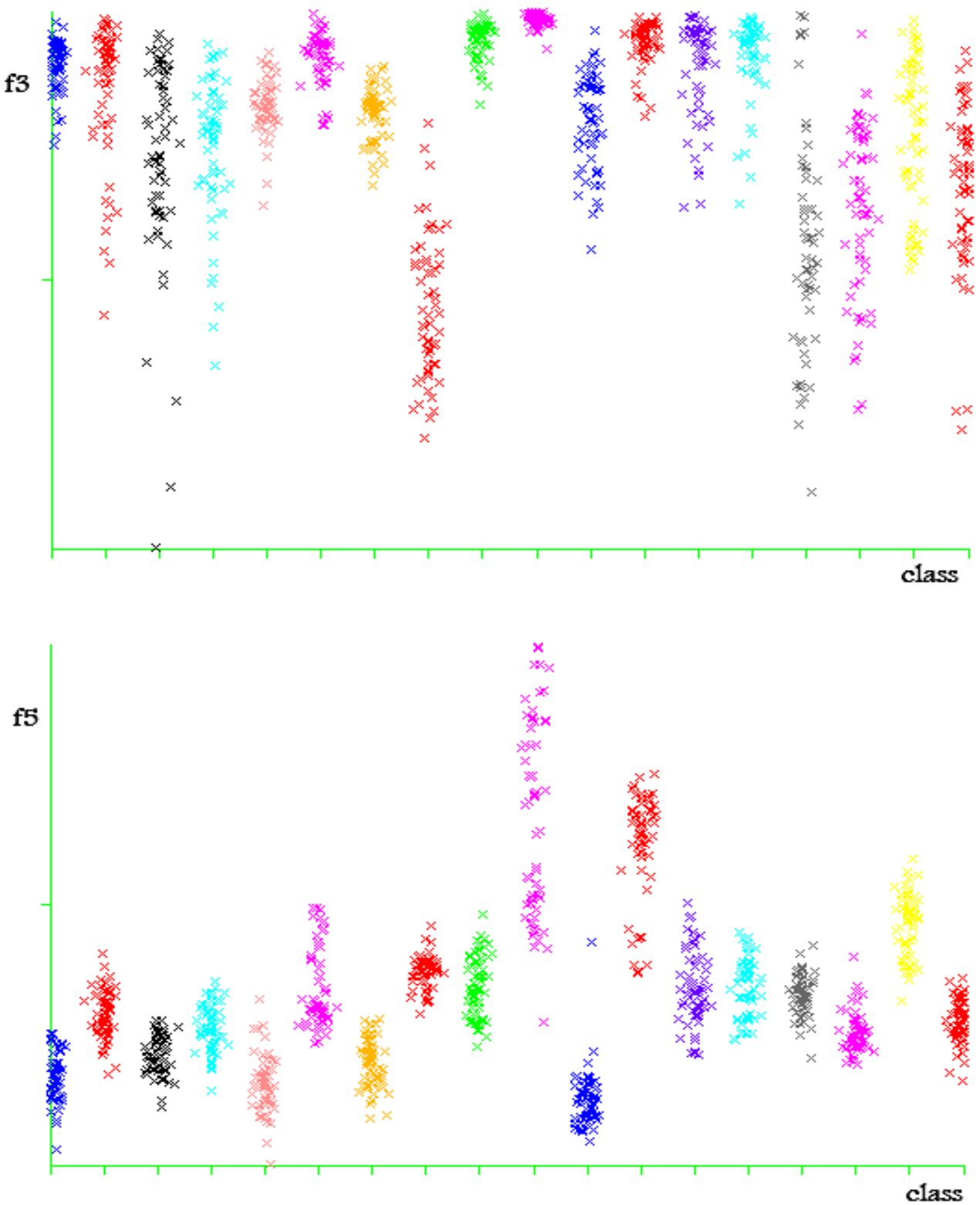

Figure 3. Some features range in each class

Although the extracted features consisting of six local descriptors and 4 global features seems to be enough good for classification of the test samples for 20 classes, it is expected using a feature reduction algorithm can lead us to a better result. Thus, a linear discriminative analysis (LDA) algorithm is employed to select the most discriminative features from the complete set of 10 features. 
Signal \& Image Processing : An International Journal (SIPIJ) Vol.2, No.3, September 2011

Finally, the selected features are used by a nearest neighbour classifier on introduced dataset to evaluate efficiency of the proposed features and algorithm. The experimental results show that attaining a recognition rate of $94.3 \%$ indicates the benefit of the method comparing to the other approaches. Furthermore, Table 1 compares the proposed method with other approaches reported for leaf recognition in different conditions such as for the leaves with hole and calamity. It shows that the proposed features and method is able to tolerate for the expecting problems with the leaves in various qualifications.

Table 1. Experimental results

\begin{tabular}{|l|l|l|l|}
\hline & Local features only & global features only & Proposed method \\
\hline Variation intensity & Do not answer & Answer & Answer \\
\hline $\begin{array}{l}\text { Leaf with hole and } \\
\text { calamity }\end{array}$ & Do not answer & Answer & Answer \\
\hline Half leaf & Answer & Do not answer & Answer \\
\hline Different season leaf & Do not answer & Answer & Answer \\
\hline
\end{tabular}

\section{CONCLUSION}

In this paper, it is shown that mixture of local descriptors and global features can be used as a feature vector for leaf recognition system where each category of the features demonstrates a different aspect of leaf properties by covering important characteristics. An important property for a leaf is veins structure where local descriptors show it with texture calculation on cooccurrence matrix. Despite all advantages of texture-based features, many leaves have simple vein so textures values are not discriminate, hence system should consider another features for describing leaves, for example shape. Many global features exist for a leaves shape that select four of them here, because these are suitable and enough for shape. Therefore, system with above features can answer very well in different conditions for different kinds. Since leaf recognition systems have to work on a large set of leaves from many different species, it is recommended applying the proposed method on a bigger data set for more assurance.

\section{REFERENCES}

[1] Ji-Xiang Du, De-Shuang Huang, Xiao-Feng Wang and Xiao Gu, (2006) "Computer-aided Plant Species Identification (CAPSI) Based on Leaf Shape Matching Technique", Transactions of the Institute of Measurement and Control.

[2] Q. Wu, Ch. Zhou, and Ch. Wang, (2006) "Feature Extraction and XML Representation of Plant Leaf for Image Retrieval", Springer, Berlin.

[3] Y. Nam, E. Hwang, and K. Byeon, (2005) “An Efficient Leaf Image Retrieval System”, Springer, Berlin.

[4] S. G. Wu, F. SH. Bao, E. Y. Xu, Y.-X. Wang, Y.-F. Chang and Q.-L. Xiang, (2007) "A Leaf Recognition Algorithm for Plant Classification Using Probabilistic Neural Network", arXiv:0707.4289v1 [cs.AI] ,29 Jul.

[5] H. Fu, Z. Chi, D. Feng, and J. Song, (2004) "Machine Learning Techniques for Ontology-based Leaf Classification", International Conference on Control, Automation, Robotics and Vision.

[6] Z. Wang, Z. Chi and D. Feng, (2003) "Shape-based Leaf Image Retrieval”, IEEE proc.-vis image process, Hong Kong. 
Signal \& Image Processing : An International Journal (SIPIJ) Vol.2, No.3, September 2011

[7] Ji-Xiang Du1,2, De-Shuang Huang1, Xiao-Feng Wang1 and Xiao Gu1, (2006) "Computer-aided Plant Species Identification (CAPSI) Based on Leaf Shape Matching Technique", Transactions of the Institute of Measurement and Control 28, 3 pp. 275_284.

[8] Ye, Y., Chen, C., Li, C.T., Fu, H., Chi, Z.: (2004) "A Computerized Plant Species Recognition System". In: Proceedings of 2004 International Symposium on Intelligent Multimedia, Video and Speech Processing, Hong Kong.

[9] Zhang, D.S., Lu, G.J.: (2004) "Review of Shape Representation and Description Techniques". Pattern Recognition 37(1), 1-19.

[10] Y. Ye, Ch. Chen, Ch.-T. Li, H. Fu, and Zh. Chi, (2004) "A Computerized Plant Species Recognition System", International Symposium on Intelligent Multimedia, Video and Speech Processing, Hong Kong.

[11] Q. Man, CH. Zheng, X. Wang, and F. Lin, (2008) "Recognition of Plant Leaves Using Support Vector Machine”, Springer, Berlin.

[12] D. G. Lowe, (1999) "Object Recognition from Local Scale-Invariant Features", International Conference on Computer Vision.

[13] X. Gu, J.-X. Du, and X.-F. Wang, (2005) "Leaf Recognition Based on the Combination of Wavelet Transform and Gaussian Interpolation", Springer, Berlin.

[14] X. Liu, K. Hou, L. Wang, P. Liu, Sichuan, (2009) "Index and Recognition for the Shape Contour of Plant Leaves", Global Congress on Intelligent Systems IEEE.

[15] S. M. Hong, B. Simpson and G. V. G. Baranoski, (2004) "Interactive Venation-based Leaf Shape Modeling", computer animation and virtual worlds 2005; 16: 415-427.

[16] J. Liu, S. Zhang, and J. Liu , (2009) “A Method of Plant Leaf Recognition Based on Locally Linear Embedding and Moving Center Hypersphere Classifier”, Springer-Verlag Berlin Heidelberg. 